\title{
Light field panorama by a plenoptic camera
}

\author{
Zhou Xue, Loic Baboulaz, Paolo Prandoni and Martin Vetterli \\ École Polytechnique Fédérale de Lausanne, Switzerland
}

\begin{abstract}
Consumer-grade plenoptic camera Lytro draws a lot of interest from both academic and industrial world. However its low resolution in both spatial and angular domain prevents it from being used for fine and detailed light field acquisition. This paper proposes to use a plenoptic camera as an image scanner and perform light field stitching to increase the size of the acquired light field data. We consider a simplified plenoptic camera model comprising a pinhole camera moving behind a thin lens. Based on this model, we describe how to perform light field acquisition and stitching under two different scenarios: by camera translation or by camera translation and rotation. In both cases, we assume the camera motion to be known. In the case of camera translation, we show how the acquired light fields should be resampled to increase the spatial range and ultimately obtain a wider field of view. In the case of camera translation and rotation, the camera motion is calculated such that the light fields can be directly stitched and extended in the angular domain. Simulation results verify our approach and demonstrate the potential of the motion model for further light field applications such as registration and super-resolution.
\end{abstract}

Keywords: Plenoptic camera, non-classical image capture, light field stitching, light field panorama

\section{INTRODUCTION}

Plenoptic cameras, also known as light-field cameras, can capture both color and geometric information of a scene in a single shot. This is achieved by recording the intensity and direction of each light ray at the same time. It enables many new imaging applications including depth estimation, digital refocusing and perspective shift. Light-field acquisitions also provide new approaches for many of the standard problems in image processing such as denoising, super-resolution, image stitching, etc.

The first consumer-grade plenoptic camera, Lytro, has generated a lot of interest when appeared in 2011. It provides a compact and portable solution for the acquisition of the light field by adding a microlens array to a conventional camera ${ }^{1}{ }^{2}$ Its major limitation however is the low resolution of the rendered images: the camera's sensor is indeed used to record both the spatial and angular information of the scene resulting in a rendered image with a resolution equal to that of the microlens array. To benefit from the great potential of light field cameras, increasing the resolution in both spatial and angular domain is a crucial step for many applications. Since increasing the resolution of the microlens array can be very expensive, taking multiple light field images and merging the data is an appealing way to tackle the problem.

Increasing the size of light field data can be achieved by aligning and stitching multiple light fields with overlapping areas for wider field of view. Birklbauer, et al. ${ }^{3}$ present the first approach to construct panorama light field image by using all-in-focus image for registration and focal-stack image for view synthesis. Our work is different from theirs in two aspects: firstly, we give a complete motion model of the light field captured by the plenoptic camera, including both camera translation and rotation. Secondly, we directly perform stitching in the 4D light field whereas both all-in-focus and focal-stack images are projections of the original light field data into low dimensions as mentioned in the work by Levin and Durand. ${ }^{4}$

This paper describes a general plenoptic camera model which is used for light fields stitching and show that classic image alignment and stitching is a sub-problem of the more general stitching problem of light fields. In the remainder of this paper, we first formalize the problem of image stitching from the perspective of light fields. We then present our acquisition and motion model of the plenoptic camera. Finally we give two applications in light field stitching based on the proposed motion model and verify the model with simulation results.

Further author information: (Send correspondence to Zhou Xue)

Zhou Xue.: E-mail: zhou.xue@epfl.ch

Computational Imaging XII, edited by Charles A. Bouman, Ken D. Sauer, Proc. of SPIE-IS\&T Electronic Imaging,

SPIE Vol. 9020, 90200S · (c) 2014 SPIE-IS\&T · CCC code: 0277-786X/14/\$18 - doi: 10.1117/12.2038254

Proc. of SPIE-IS\&T/ Vol. 9020 90200S-1 


\section{IMAGE STITCHING IN LIGHT FIELDS}

\subsection{Background on light fields}

In this paper, we use a two plane parameterization to represent the light field in which each light ray in the space is described by using its intersection with two parallel planes, the image plane $(p, q)$ and camera plane

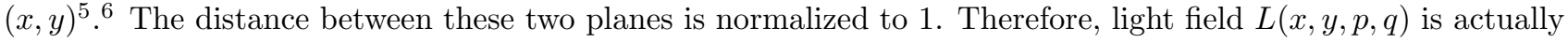
the radiance intensity of the light ray intersecting with $(x, y)$ plane from the $(p, q)$ direction. Without loss of generality, we perform the analysis on a 2D light field $L(x, p)$ by fixing $y$ and $q$ for the sake of simplicity.

Since the light field is indeed an intensity function of light rays, the ray transfer matrix analysis is used in this paper to describe the linear transformation of the light field. The two fundamental ray transformations, ray propagation in space $\boldsymbol{A}_{p}$ and refraction $\boldsymbol{A}_{f}$ by a lens are described respectively as follows:

$$
\boldsymbol{A}_{p}=\left[\begin{array}{cc}
1 & p \\
0 & 1
\end{array}\right], \quad \boldsymbol{A}_{f}=\left[\begin{array}{cc}
1 & 0 \\
-f^{-1} & 1
\end{array}\right]
$$

where $p$ denotes the propagating distance and $f$ is the lens' focal length.

A simple pinhole camera model is used to demonstrate the relation between the light field and captured images. As shown in Figure 1, an example of 2D light field is generated by using a moving pinhole camera to capture one horizontal line from an oil painting. For the sake of simplicity, the oil painting is modeled as a fronto-parallel plane $Z$ meters away from the camera with Lambertian-reflectance properties. The moving camera is on the plane $x$ and each image is defined on the plane $p$ relative to the camera position.

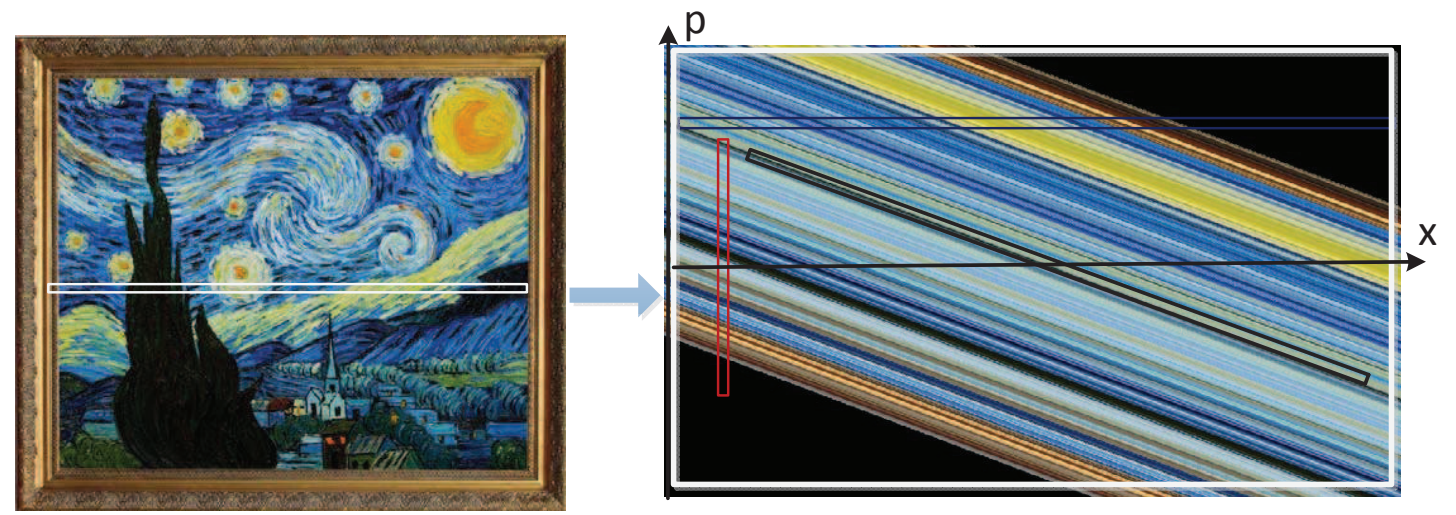

Figure 1: An example of a 2D light field is generated from one horizontal line in a fronto-parallel oil painting with Lambertian-reflectance properties. In the $2 \mathrm{D}$ light field on the right, the red slice denotes an image captured by a pinhole camera on the camera plane; the blue slice denotes a bundle of parallel light rays captured theoretically by a camera at infinity; the black slice denotes an image taken by a pinhole camera placed on the oil painting.

The red slice in the 2D light field represents an image taken by the moving pinhole camera on the camera plane $x$. All the light rays within the slice converge on the plane $x$. The blue slice represents a bundle of parallel light rays which can theoretically be captured by a pinhole camera at infinity.

Another special slice is represented by the black slice whose slope is $-1 / Z$ and $Z$ is the distance between the oil painting and the camera plane $x$. The light rays within the green slice all converge at $Z$ meters in front of the camera plane $x$ and they are exactly from the same spot on the surface of the oil painting. Since the oil painting is assumed have Lambertian-reflectance properties, the intensities within the black slice remain constant for all the light rays from different directions. Theoretically, the black slice is captured by a pinhole camera positioned on the oil painting.

In conclusion, slices in the light field with different slopes are images which can be virtually captured by pinhole cameras at planes away from the original camera plane $x$. A slice in $2 \mathrm{D}$ light field with a slope $1 / z$ can 
be seen as equivalent to an image captured by a pinhole camera positioned at $z$ meters away from the original camera plane $x$. A positive $z$ indicates that the position is in front of the camera plane whereas a negative $z$ indicates that the position is behind the camera plane. Two extreme cases are vertical slices and horizontal slices which are images captured by camera on plane $x$ and at infinity respectively.

Last but not the least, $p$ represents the image coordinate normalized by the focal length of the pinhole camera. The range of the light field slice in the $p$ dimension remains constant for the same camera. Slices with negative slopes are images with magnified texture of the oil painting whereas the ones with positive slopes are images with shrunken texture.

\subsection{Understanding image stitching in light fields}

Aligning images and stitching them into wide-angle panoramas are among the most widely used algorithms in computer vision. A practical stitching algorithm requires robust solutions for image alignment, warping, artifacts removal, etc. In this paper, we only focus on the mathematical model relating pixel coordinates in one image to pixel coordinates in another ( $\mathrm{see}^{7}$ for an excellent survey). A pinhole camera model is also used for the sake of simplicity.
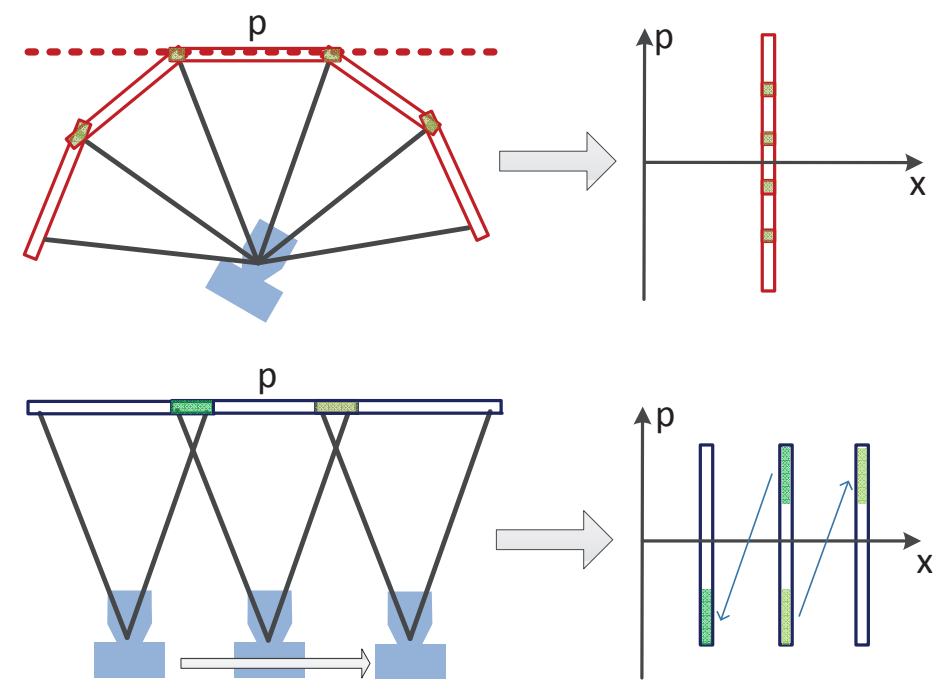

Figure 2: Image stitching with camera translation or rotation on the left and their representation in 2D light field on the right. The red slices on the top row denote the camera rotation whereas the blue ones on the bottom denote the camera translation. The green areas are used to represent overlapping areas among capture images.

A stitched panorama is usually made by joining multiple photographs with slightly overlapping fields of view as shown in Figure 2 on the left. These images could either be taken by camera rotations around camera's optical center or camera translations parallel to the image plane.

\subsubsection{Creating panorama by camera rotations}

To describe the motion model of light fields, we consider a pinhole camera capturing a 1D image in the 2D light field $L(x, p)$. Camera rotations around the optical center in $3 \mathrm{D}$ are reduced to a tilt of the pinhole camera around its optical center for 1D images. The image captured by a pinhole camera can thus be represented as follows:

$$
I(p)=L\left(x_{0}, p\right), \quad p \in\left[-\frac{S}{2 f}, \frac{S}{2 f}\right]
$$

where $x_{0}$ represents the location of the camera, $S$ represents the size of the camera sensor and $f$ represents the focal length of the pinhole camera. The original image $I(p)$ and the image $I_{r}(p)$ after camera rotation satisfy $I_{r}(\tan (\arctan (p)-\theta))=I(p)$ where $\theta$ is the rotation angle. 


\subsubsection{Creating panorama by camera translations}

As for the images captured by camera translations, the 1D images are slices of the light field at different camera locations such as $L\left(x_{0}, p\right)$ and $L\left(x_{0}^{\prime}, p\right)$ where $p \in\left[-\frac{S}{2 f}, \frac{S}{2 f}\right]$. Under the assumption that the captured scene is a fronto-parallel Lambertian surface, the relation between two images can be represented as

$$
I(p)=I^{\prime}(p+\Delta p), \quad \Delta p=\left(x_{0}-x_{0}^{\prime}\right) \frac{f}{Z}
$$

where $Z$ is the depth of the scene.

As shown on the right of Figure 2, image stitching by camera rotations and translations can both be described in the light field. By rotating the camera, acquired images are shifted in the $p$ dimension and a wider field of view is achieved by merging these images directly. By translating the camera, multiple images in the $x$ dimension can be seen as equivalent to multiple images in the $p$ dimension when the captured scene is a planar surface with Lambertian-reflectance properties. In conclusion, image stitching mainly focuses on merging multiple slices in the light field for a wider field of view in the $p$ dimension. However, light field stitching must consider both $x$ and $p$ dimensions which makes it a more general problem.

\section{MOTION MODEL FOR LIGHT FIELD STITCHING}

\subsection{Acquisition model of plenoptic camera}

We consider a simplified plenoptic camera model comprising a pinhole camera moving behind a thin lens of focal length $f$. The distance between the pinhole camera plane and the main lens is defined as $b$. Then the focused plane of the main lens is $a$ meters away which satisfies the thin lens equation $1 / f=1 / a+1 / b$.

We denote by $\mathbf{L}[m, n]$ the discrete $2 \mathrm{D}$ light field data captured by a plenoptic camera where $m$ and $n$ respectively stand for the position of the pinhole camera and the pixel index within a pinhole camera image. The 1D image obtained by fixing $m$ is commonly referred as the pinhole camera image whereas the 1D image obtained by fixing $n$ is referred as the sub-aperture image. Then the imaging process shown in Figure 3 can be described as follows:

$$
\begin{aligned}
\mathbf{L}[m, n] & =\iint L(x, p) \phi\left(x / T_{x}-m, p / T_{p}-n\right) \mathrm{d} x \mathrm{~d} p \\
& =\left\langle L(x, p), \phi\left(x / T_{x}-m, p / T_{p}-n\right)\right\rangle
\end{aligned}
$$

where $T_{x}$ and $T_{p}$ represent the sampling periods on $x, p \in \mathrm{R},[m, n] \in \mathrm{Z}$ represent the pixel index of the acquired light field data and $\phi$ represents the sampling kernel in the light field.

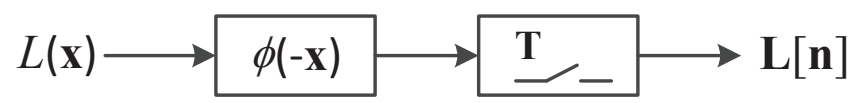

Figure 3: Sampling process of a plenoptic camera.

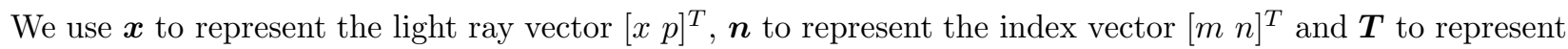
the sampling periods $\operatorname{diag}\left(T_{x}, T_{p}\right)$ for the sake of simplicity. By assuming a perfect optical system, the point spread function of the main lens becomes a Dirac function. Each pixel of the pinhole camera is assumed to be infinitely small and free of noise. Then we can rewrite the sampling equation Equation (4) as follows:

$$
\begin{aligned}
\mathbf{L}[\boldsymbol{n}] & =\left\langle L(x, p), \delta\left(x / T_{x}-m, p / T_{p}-n\right) * \phi(x, p)\right\rangle \\
& =\left\langle L(x, p), \delta_{m T_{x}, n T_{p}}(x, p)\right\rangle \\
& =\left\langle L(\boldsymbol{x}), \delta_{\boldsymbol{T} \cdot \boldsymbol{n}}(\boldsymbol{x})\right\rangle .
\end{aligned}
$$

As described in Equation (5), each pixel of the pinhole camera captures one light ray emitted from the scene. The discrete light field $\mathbf{L}[m, n]$ can be directly related to the continuous light field $L(\boldsymbol{x})$. 
To get the exact values of the sampling periods, we introduce the light field $L^{\prime}(x, p)$ defined on the plane of the moving pinhole camera as shown in Figure 4. The sampling periods on $L^{\prime}(x, p)$ are directly defined by the pinhole camera's motion and its sensor resolution. The spatial sampling period $T_{x}$ is the moving step size of the pinhole camera and the angular sampling period $T_{p}$ is the pixel size normalized by pinhole camera's focal length.

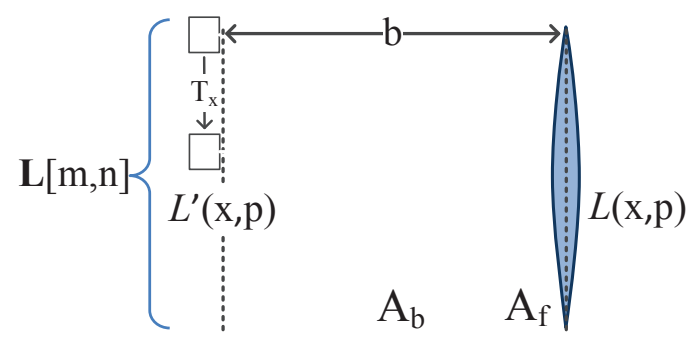

Figure 4: An illustration of the simplified plenoptic camera. A pinhole camera moves behind a thin lenswith a step size of $T_{x}$. The light field $L(x, p)$ is defined on the main lens plane whereas $L^{\prime}(x, p)$ is defined on the pinhole camera plane. The transformation between $L(x, p)$ and $L^{\prime}(x, p)$ is a combination of the refraction $A_{f}$ and the propagation $A_{b}$.

By using Equation (1), the transformation between the light field $L(x, p)$ and $L^{\prime}(x, p)$ is a combination of a refraction $\boldsymbol{A}_{f}$ by the main lens and a $b$-meter propagation $\boldsymbol{A}_{b}$ as follows:

$$
\boldsymbol{A}=\boldsymbol{A}_{b} \boldsymbol{A}_{f}=\left[\begin{array}{ll}
1 & b \\
0 & 1
\end{array}\right]\left[\begin{array}{cc}
1 & 0 \\
-f^{-1} & 1
\end{array}\right]
$$

Therefore, we have $L^{\prime}(\boldsymbol{x})=L\left(\boldsymbol{A}^{-1} \boldsymbol{x}\right)$ from which we can estimate the sampling periods on $L(\boldsymbol{x})$ as follows:

$$
\begin{aligned}
\mathbf{L}[\boldsymbol{n}] & =\left\langle L^{\prime}(\boldsymbol{x}), \delta_{\boldsymbol{T} \cdot \boldsymbol{n}}(\boldsymbol{x})\right\rangle \\
& =\left\langle L\left(\boldsymbol{A}^{-1} \boldsymbol{x}\right), \delta_{\boldsymbol{T} \cdot \boldsymbol{n}}(\boldsymbol{x})\right\rangle \\
& =\left\langle L(\boldsymbol{x}), \delta_{\boldsymbol{T} \cdot \boldsymbol{n}}(\boldsymbol{A} \boldsymbol{x})\right\rangle \\
& =\left\langle L(\boldsymbol{x}), \delta_{\boldsymbol{A}^{-1} \boldsymbol{T} \cdot \boldsymbol{n}}(\boldsymbol{x})\right\rangle .
\end{aligned}
$$

In Equation (7), we used $\boldsymbol{T}=\operatorname{diag}\left(T_{x}, T_{p}\right)$ to represent the sampling periods on the plane of the moving pinhole camera where $T_{x}$ and $T_{p}$ are defined by the pinhole camera's moving step size and normalized pixel size respectively. By using Equation (6), the sampling periods on $L(\boldsymbol{x})$ which is defined on the main lens plane can be calculated as follows:

$$
\begin{aligned}
\boldsymbol{A}^{-1} \boldsymbol{T} & =\left[\begin{array}{cc}
1 & -b \\
f^{-1} & 1-b f^{-1}
\end{array}\right]\left[\begin{array}{cc}
T_{x} & 0 \\
0 & T_{p}
\end{array}\right] \\
& =\left[\begin{array}{cc}
T_{x} & -b T_{p} \\
f^{-1} T_{x} & -b a^{-1} T_{p}
\end{array}\right] .
\end{aligned}
$$

The columns of the matrix $\boldsymbol{A}^{-1} \boldsymbol{T}$ are the sampling periods pinhole-camera-wise and pixel-wise respectively. The first column $\left[\begin{array}{ll}T_{x} & f^{-1} T_{x}\end{array}\right]^{T}$ determines the distance between pixels within the same pinhole camera and the second column $\left[b T_{p}-b a^{-1} T_{p}\right]^{T}$ determines the distance between the same pixel under two consecutive pinhole cameras in the light field. Therefore, the rectangular lattice on $L^{\prime}(x, p)$ becomes a parallelogram lattice on $L(x, p)$ as shown in Figure 5 . The parallelogram-shaped sampling pattern is specified by the camera parameters.

\subsection{Translation model of light fields}

We first describe the motion model by only considering camera translations, namely when the plenoptic camera is shifted in a plane parallel to the main lens and pinhole camera plane. The $2 \mathrm{D}$ light field data $L_{0}(x, p)$ and 

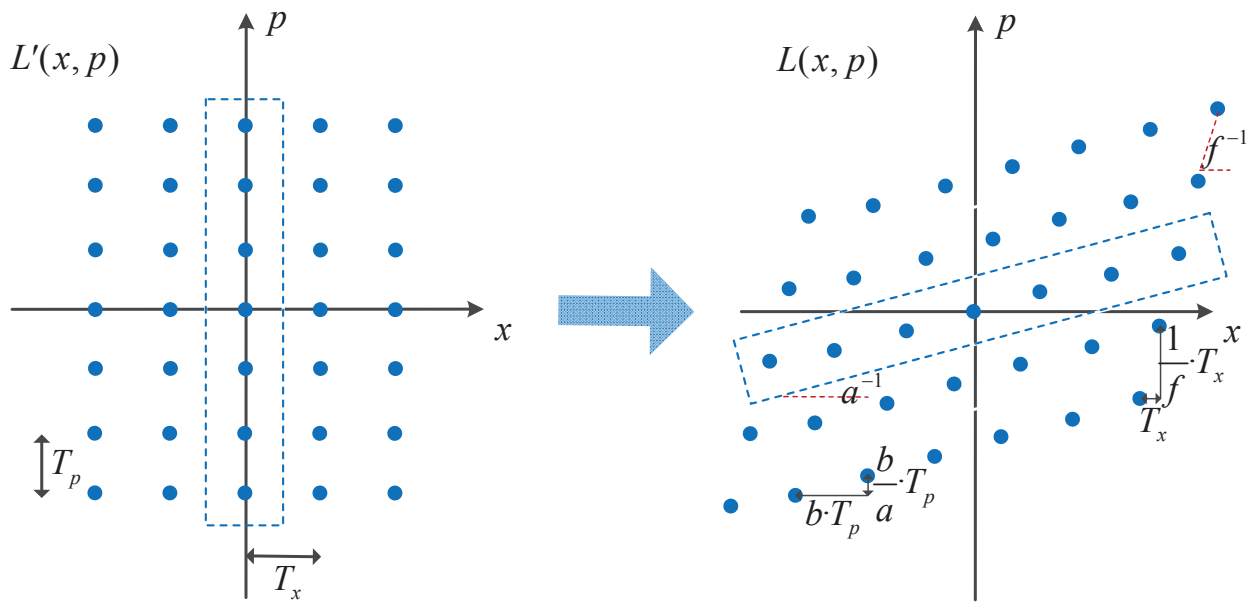

Figure 5: Sampling operator of plenoptic camera on $L^{\prime}(x, p)$ and $L(x, p)$. The pixels from the same pinhole camera are shown inside the dashed rectangles.

$L_{1}(x+\Delta x, p)$ are acquired by moving the plenoptic camera for $\Delta x$ meters in world coordinates. Then the discrete translation $(\Delta m, \Delta n)$ between these two acquired light fields is as follows:

$$
\mathbf{L}_{0}[m, n]=\mathbf{L}_{1}[m+\Delta m, n+\Delta n] .
$$

By using the sampling period $\boldsymbol{A}^{-1} \boldsymbol{T}$ from Equation (6), we can relate the camera translation to the pixel shift in the discrete light field as follows:

$$
\begin{aligned}
\boldsymbol{A}^{-1} \boldsymbol{T}\left[\begin{array}{l}
m \\
n
\end{array}\right] & =\left[\begin{array}{l}
x \\
p
\end{array}\right] \\
\boldsymbol{A}^{-1} \boldsymbol{T}\left[\begin{array}{c}
m+\Delta m \\
n+\Delta n
\end{array}\right] & =\left[\begin{array}{c}
x+\Delta x \\
p
\end{array}\right]
\end{aligned}
$$

which can be further simplified by removing the common terms to derive

$$
\boldsymbol{A}^{-1} \boldsymbol{T}\left[\begin{array}{l}
\Delta m \\
\Delta n
\end{array}\right]=\left[\begin{array}{c}
\Delta x \\
0
\end{array}\right]
$$

which also leads to the linear relation between $\Delta m$ and $\Delta n$ as follows:

$$
\Delta n=\frac{a}{f \cdot b} \frac{T_{x}}{T_{p}} \Delta m .
$$

Therefore, the 2D camera translation results in a $4 \mathrm{D}$ shift in the discrete light field data with only 2 degrees of freedom. The ratio between the pixel shift in angular and spatial dimension is determined by the specific parameters of the plenoptic camera as shown in Equation (11).

\subsection{Rotation model of light fields}

In this section, we describe the light field motion model by only considering camera rotations around the main lens' optical center. A 2D example is used to show how a plenoptic camera is rotated around its optical center by an angle $\phi$ clockwise in Figure 6. The blue line with two arrows represents the main lens of the original plenoptic camera whereas the green one represents the main lens after rotation. The dashed lines denote the same light ray defined on these two main lens planes. The variable $L_{r}\left(x_{r}, p_{r}\right)$ is used for the light field after rotation to distinguish it from the original light field $L(x, p)$. 

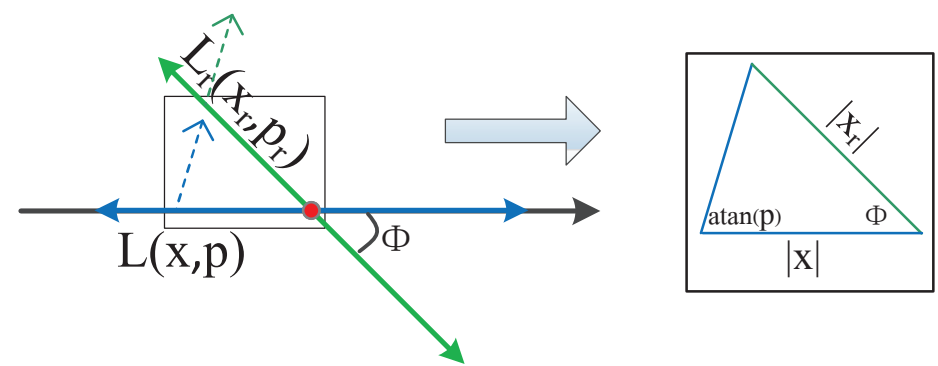

Figure 6: The plenoptic camera is rotated by $\phi$ around the optical center of its main lens. The dashed lines $L(x, p)$ and $L_{r}\left(x_{r}, p_{r}\right)$ denote the same light ray captured by the plenoptic camera before and after rotation. The geometric relations between $\left(x_{r}, p_{r}\right)$ and $(x, p)$ are shown on the left.

By using geometric relations between $\left(x_{r}, p_{r}\right)$ and $(x, p)$, we conclude that

$$
\begin{aligned}
p & =\frac{p_{r}-\tan \phi}{1+p_{r} \tan \phi}=\tan \left(\arctan \left(p_{r}\right)-\phi\right) \\
x & =x_{r} \sin \phi \cdot p+x_{r} \cos \phi
\end{aligned}
$$

In Equation (12), we observe that the light field transformation by camera rotation is not a linear operation in the $p$ dimension. As for the transformation in the $x$ dimension, the shift depends on both the camera rotation and the direction $p$ of the light ray. Only when the rotation angle $\phi$ is very small, then the light field transformation by camera rotation can be seen approximately as a linear transformation. Therefore, extending the light field in $p$ dimension by camera rotation can only work for small rotation angles. As $\phi$ increases, the parallelogram shape of the sampling pattern after rotation cannot hold when aligning to the original light field. Therefore, we don't consider light field stitching purely by camera rotations.

\section{EXPERIMENTS AND DISCUSSIONS}

Based on the proposed motion model, we use the plenoptic camera as an image scanner and perform light field stitching to increase the size of the acquired light field data. We report two applications in light field stitching by camera translations and a combination of camera translations and rotations. Our main goal here is to verify the motion model and demonstrate its potential for light field acquisition.

\subsection{Light field stitching by camera translation}

By using Equation (7), we establish the relations between the camera motion and the acquired light field data defined by the pinhole camera index $m$ and the pixel index $n$. Then we can simply capture multiple light fields without overlapping areas as shown in Figure 7. A direct stitching approach is demonstrated with the blue dashed parallelogram. Each pinhole camera image is extended at the cost of reducing the size of the sub-aperture image (same pixel index in all pinhole cameras). The reduction in spatial range is actually due to the fact that the captured data has the shape of a parallelogram in the $2 \mathrm{D}$ light field defined on the main lens plane of the plenoptic camera.

To avoid reducing the size of the sub-aperture image, we propose to resample the captured light field to increase the field of view. As mentioned in Section 2.1, slices with different slopes are actually images captured virtually by cameras at different planes. By carefully choosing the slicing slopes in the light field, the sub-aperture image represented with the blue dashed line obtains a wider field of view as shown in Figure 8.

Based on the strategy in Figure 8, a virtual plenoptic camera in Matlab is used to capture multiple light fields at a close distance to a slanted plane painted with pink circles. The texture is chosen to be a band-limited signal. Since the texture is painted on a slanted plane, the light field is also a band-limited signal as shown in. ${ }^{8}$ The Nyquist condition is satisfied by the sampling periods in the $4 \mathrm{D}$ light field. The dimension $\left(n_{p}, n_{q}, m_{x}, m_{y}\right)$ of each acquired light field data is $21 \times 21 \times 15 \times 15$. A total number of $31 \times 31$ light field acquisitions are used for light field stitching. One stitched sub-aperture image of the final panorama light field is shown in Figure 9 with a resolution of $(31 \times 21) \times(31 \times 21)$. 


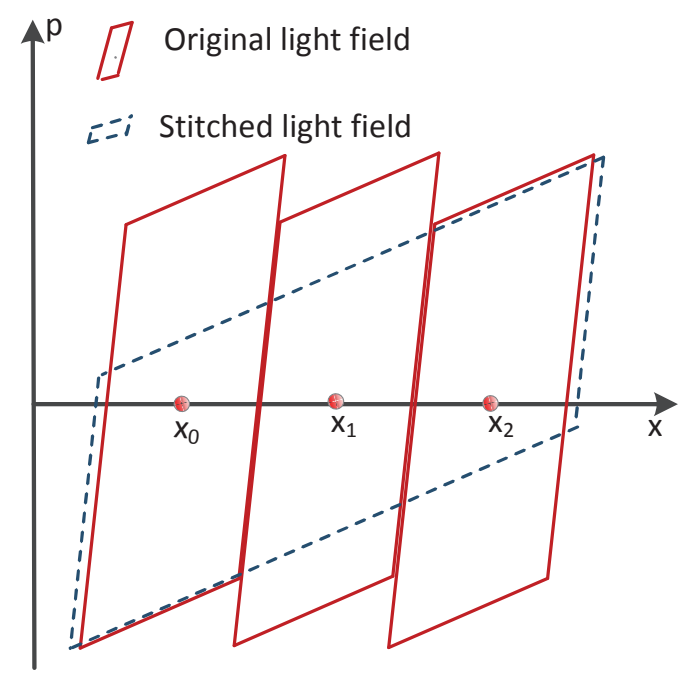

Figure 7: The red parallelograms represent the light field taken by plenoptic cameras at $x_{0}, x_{1}$ and $x_{2}$ and the blue parallelogram with dashed line represents a direct stitched light field by extending the pinhole camera image.

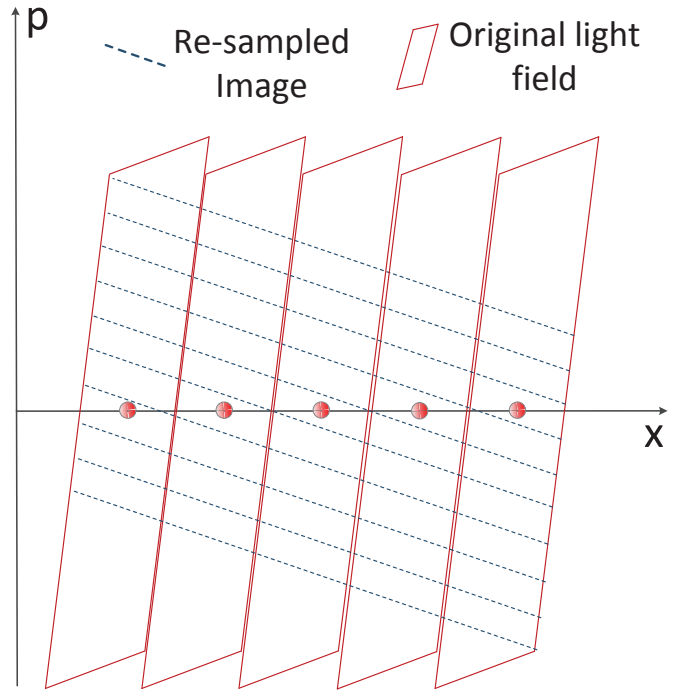

Figure 8: Re-sample multiple light field samples to create a virtual light field image taken behind the camera plane.

\subsection{Light Field Stitching with Camera Rotation and Translation}

We can also perform light field stitching by combining camera rotations and translations. Therefore, we can extend each pinhole image directly without resampling.

As shown in Figure 7, pinhole camera images at the boundaries of the light field acquisition cannot be extended because of the parallelogram shape. By rotating the plenoptic camera, acquired light fields are shifted in $p$ dimension in order to extend each pinhole camera image as shown in Figure10.

The simulation results of 2D light field stitching by both camera rotations and translations are given in Figure 10. Each plenoptic camera with different translation is rotated around the optical center of its main lens. The specific camera parameters for the simulation is shown in Table 1. The parameters are similar to the ones of a Lytro.

Then we first use Equation (6) to calculate the range of the each pinhole camera image in $x$ dimension as 

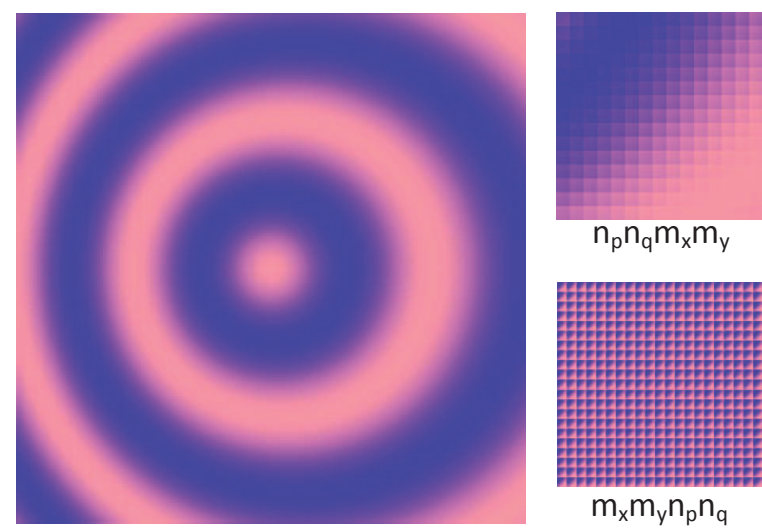

$m_{x} m_{y} n_{p} n_{q}$

Figure 9: A panorama image created from $31 \times 31$ light field images. One sub-aperture image of the final panorama light field is on the left and one acquired light field image with different display orders $\left(n_{p}, n_{q}, m_{x}, m_{y}\right)$ (an array of microlens image) and $\left(m_{x}, m_{y}, n_{p}, n_{q}\right)$ (an array of sub-aperture image) is on the right.

\begin{tabular}{|c|c|c|c|}
\hline \multicolumn{4}{|c|}{ Plenoptic camera parameters for simulation } \\
\hline \multirow{2}{*}{ Main Lens } & $\mathrm{f}$ & $\mathrm{a}$ & $\mathrm{b}$ \\
\cline { 2 - 4 } & $6.45 \mathrm{~mm}$ & $0.3 \mathrm{~m}$ & $6.9 \mathrm{~mm}$ \\
\hline \multirow{2}{*}{ Microlens Array } & pitch & $\mathrm{f}$ & $\mathrm{b}$ \\
\cline { 2 - 4 } & $1.4 \times 10^{-5} \mathrm{~m}$ & $2.5 \times 10^{-3} \mathrm{~mm}$ & $2.5 \times 10^{-3} \mathrm{~mm}$ \\
\hline \multirow{2}{*}{ Sensor } & pitch & angular dimension & spatial dimension \\
\cline { 2 - 4 } & $1.4 \times 10^{-6} \mathrm{~m}$ & $11 \times 11$ & $301 \times 301$ \\
\hline
\end{tabular}

Table 1: Specific camera paramters for light field stitching simulation.

follows:

$$
\Delta x=N \cdot T_{p} \cdot b
$$

where $N$ represents the size of each pinhole camera image, $T_{p}$ represents the sampling period in $p$ for each pixel and $b$ represents the distance between the main lens and the pinhole camera plane. Then the camera is translated for $\Delta x$ to maximize the stitched range in $x$.

As shown in Figure 5, each pinhole camera image is a slice in the light field with a slope $1 / a$. To compensate for the parallelogram shape, each pinhole camera image should be shifted by $\Delta x / a$ in the $p$ dimension. By using Equation (12), the plenoptic camera is rotated around the main lens' optical center by an angle $\Phi$ as follows:

$$
\Phi=\arctan \left(\frac{\Delta x}{a}\right)
$$

which is an approximation by assuming the original $p$ to be zero.

As shown in Figure 10, five light field images are generated with different translations $(-2 \Delta x,-\Delta x, 0, \Delta x$ and $2 \Delta x$ ). To compensate for the shift in $p$ dimension, each camera is rotated for an angle calculated by Equation (15). From the simulation results in Figure 10, we can observe that the required range of the light field to extend each pinhole camera image is covered by the acquired data. Further treatment for interpolation are also needed especially when $\phi$ increases.

In conclusion, light field stitching by camera rotations and translations effectively increases the size of each pinhole camera image of the light field. However, camera rotations are usually more difficult to implement. Due to non-linearity of the light field transformation by camera rotation, this stitching method only works effectively for when the rotation angle $\phi$ is small. 


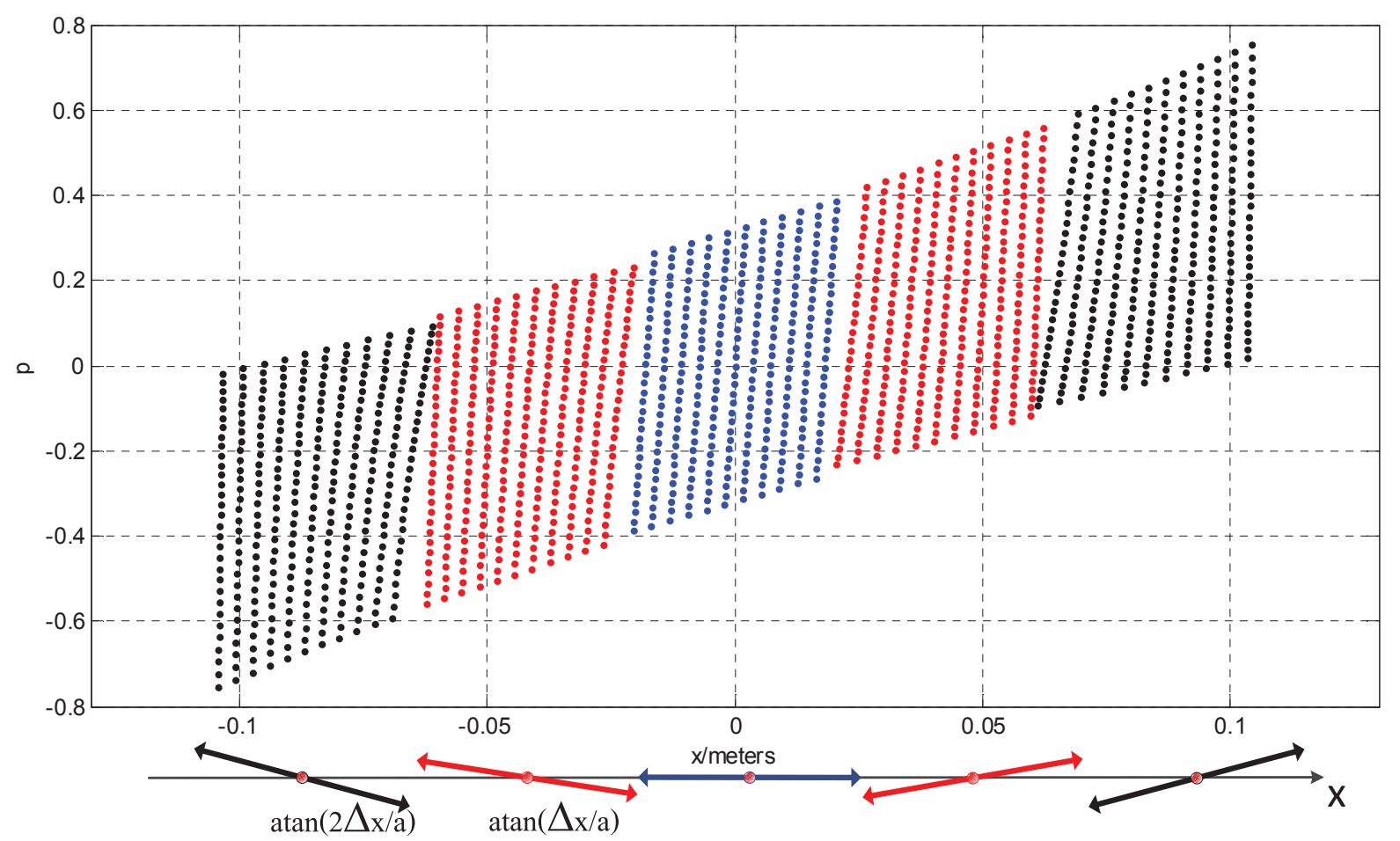

Figure 10: Five plenoptic cameras with different translations $(-2 \Delta x,-\Delta x, 0, \Delta x, 2 \Delta x)$ and rotations are used to sample the light field. As $\phi$ increases, the distortion of the parallelogram shape becomes more obvious.

\subsection{Conclusion and future work}

In this paper, we propose a motion model of a simplified plenoptic camera and present its applications in light field stitching. The plenoptic camera is used as a scanner and multiple images are merged into one light field with larger spatial range or angular range.

Firstly, light fields by camera translations are resampled to create light field panoramas. By slicing the acquired multiple light fields with different slopes, each sub-aperture image of the new light field has a wider field of view.

Secondly, by combining camera rotations and translations, the angular range of the light field can be increased directly. The motion model of camera rotation is described and verified by the simulation of a $2 \mathrm{D}$ light field which can be easily extended to $4 \mathrm{D}$. But this type of acquisition is limited to a certain angular range because of the non-linearity of the light field transformation by camera rotation.

In the future, we will extend our work to light field registration and super-resolution based on the proposed motion model.

\section{ACKNOWLEDGMENTS}

This research is being supported by Google Focus Award-eFacsimile research, SNSF grant number 200021_138081 and ERC Advanced Investigators Grant: Sparse Sampling: Theory, Algorithms and Applications SPARSAM no. 247006.

\section{REFERENCES}

[1] Ng, R., "Fourier slice photography," in [ACM SIGGRAPH 2005 Papers], SIGGRAPH '05, 735-744, ACM, New York, NY, USA (2005).

[2] Lumsdaine, A. and Georgiev, T., "The focused plenoptic camera," in [In Proc. IEEE ICCP], 1-8 (2009). 
[3] Birklbauer, C. and Bimber, O., "Panorama light-field imaging.," in [SIGGRAPH Posters], ACM (2012).

[4] Levin, A. and Durand, F., "Linear view synthesis using a dimensionality gap light field prior," in [In Proc. IEEE CVPR], 1-8 (2010).

[5] Gortler, S. J., Grzeszczuk, R., Szeliski, R., and Cohen, M. F., "The lumigraph," in [Proceedings of the 23rd Annual Conference on Computer Graphics and Interactive Techniques], SIGGRAPH '96, 43-54, ACM, New York, NY, USA (1996).

[6] Levoy, M. and Hanrahan, P., "Light field rendering," in [Proceedings of the 23rd Annual Conference on Computer Graphics and Interactive Techniques], SIGGRAPH '96, 31-42, ACM, New York, NY, USA (1996).

[7] Szeliski, R., "Image alignment and stitching: A tutorial," Found. Trends. Comput. Graph. Vis. 2, 1-104 (Jan. 2006).

[8] Do, M. N., Marchand-Maillet, D., and Vetterli, M., "On the bandwidth of the plenoptic function," Trans. Img. Proc. 21, 708-717 (Feb. 2012). 